\title{
Curative and organ-preserving treatment with intra-arterial carboplatin induction followed by surgery and/or radiotherapy for advanced head and neck cancer: single-center five-year results Giulia Bertino*1, Marco Benazzo1, Patrizia Gatti1, Gianni Bernardo², Franco Corbella ${ }^{3}$, Carmine Tinelli ${ }^{4}$, Federico Zappoli ${ }^{5}$ and Eugenio Mira ${ }^{1}$
}

\author{
Address: ${ }^{1}$ Department of Otolaryngology, University of Pavia - IRCCS Policlinico S Matteo, Pavia, Italy, ${ }^{2}$ Department of Oncology, IRCCS \\ Fondazione "Salvatore Maugeri", Pavia, Italy, ${ }^{3}$ Department of Radiation Oncology, IRCCS Policlinico S. Matteo, Pavia, Italy, ${ }^{4}$ Biometrics Unit, \\ IRCCS Policlinico S. Matteo, Pavia, Italy and ${ }^{5}$ Radiodiagnostic Unit, IRCCS Policlinico S. Matteo, Pavia, Italy \\ Email: Giulia Bertino* - giulia.bertino@tin.it; Marco Benazzo - m.benazzo@smatteo.pv.it; Patrizia Gatti - gatti_patrizia@libero.it; \\ Gianni Bernardo - gbernardo@fsm.it; Franco Corbella - f.corbella@smatteo.pv.it; Carmine Tinelli - c.tinelli@smatteo.pv.it; \\ Federico Zappoli - f.zappoli@smatteo.pv.it; Eugenio Mira - e.mira@smatteo.pv.it \\ * Corresponding author
}

Published: II April 2007

BMC Cancer 2007, 7:62 doi:10.1 186/147/-2407-7-62

This article is available from: http://www.biomedcentral.com/I47I-2407/7/62

(C) 2007 Bertino et al; licensee BioMed Central Ltd.

This is an Open Access article distributed under the terms of the Creative Commons Attribution License (http://creativecommons.org/licenses/by/2.0), which permits unrestricted use, distribution, and reproduction in any medium, provided the original work is properly cited.
Received: 22 November 2006

Accepted: II April 2007

\begin{abstract}
Background: This study evaluated the feasibility, toxicity, response rate and survival of neoadjuvant superselective intra-arterial infusion of high dose carboplatin in advanced head and neck cancer.

Methods: Forty-six patients with primary head and neck squamous cell carcinoma received 3 cycles of intra-arterial carboplatin (300 to $350 \mathrm{mg} / \mathrm{m}^{2}$ per cycle every 2 weeks), followed by radiotherapy or surgery plus radiotherapy.

Results: No complications or severe toxicity occurred. Sixteen patients (35\%) were complete responders, $20(43 \%)$ partial responders while 10 (22\%) did not respond to treatment. After completion of the multimodality treatment, $38 / 46$ patients $(83 \%)$ were complete responders. After a 5 -year follow-up period, I8/46 patients (39\%) are alive and disease-free, $3(6,5 \%)$ have died of a second primary tumor and $25(54,5 \%)$ have died of the disease.

Conclusion: Intra-arterial carboplatin induction chemotherapy is a safe, well-tolerated technique that discriminates between responders and non-responders and so may have prognostic significance in planning further integrated treatments aimed to organ preservation for advanced head and neck carcinomas.
\end{abstract}

\section{Background}

In recent years chemotherapy has become an essential part of multimodality curative treatments for advanced head and neck cancer, with prolongation of survival and better organ preservation [1-3]. Several strategies have been developed to increase the efficacy of these integrated treatment protocols while decreasing their associated toxicity, e.g. induction or neoadjuvant versus concomitant chemotherapy, intra-arterial versus intravenous systemic administration of the drugs, single or multiple combined chemotherapeutic agents. 
According to the detailed meta-analysis by Pignon et al. [1], today the gold standard therapy for patients with locally advanced head and neck squamous cell carcinoma seems to be concurrent chemotherapy-radiation treatment, with an absolute improvement of $8 \%$ in five yearsurvival compared to other protocols. However, some reports suggest that also induction chemotherapy may improve survival, locoregional control and organ preservation in oropharyngeal and laryngeal cancer [4-8]. According to Al Sarraf further advantages obtained by giving induction chemotherapy first, the incidence of systemic micrometastases is reduced, the cancer is downstaged in approximately $90 \%$ of the cases and up $50 \%$ of patients may achieve a complete clinical response [2].

A large body of evidence supports the theoretical attractiveness of intra-arterial chemotherapy, related to the first pass of the drug through the tumor bed and to the possibility of increasing the doses of the chemotherapeutic agent, thus minimizing systemic toxic side effects [9].

Compared to intravenous systemic chemotherapy protocols, intra-arterial regimens are based on the use of a single chemotherapeutic agent, namely cisplatin. Currently, platinum derivatives are the most effective drugs in the treatment of squamous cell carcinoma of the head and neck, and the second-generation platinum drug, carboplatin, possesses all of the radiopotentiation characteristics of cisplatin with less toxic side effects $[2,10]$.
Over recent years our group has performed a pilot study of high-dose intra-arterial carboplatin induction chemotherapy on patients with untreated advanced squamous cell carcinoma of the upper aerodigestive tract. Neoadjuvant intra-arterial chemotherapy was part of a multimodality regimen combining radiotherapy in responder patients, surgery plus radiotherapy in resectable non-responder patients and palliative radiotherapy in non-resectable non-responder patients [11]. The purpose of the present study was to assess the feasibility and technical problems of intra-arterial transfemoral superselective perfusion, the maximum-tolerated dose of carboplatin, systemic and local toxicity and the response rate after induction chemotherapy on the same enlarged population after a longer term follow-up. After completing the therapeutic options built into our multimodality protocol, we assessed fiveyear overall and disease-free survival rates in patients.

\section{Methods \\ Patient characteristics}

Forty-six patients (43 men and 3 women), aged from 39 to 75 years (mean 58,3, median 42,1 ), with previously untreated squamous cell carcinoma of the upper aerodigestive tract were treated between November 1993 and December 2000. The pre-treatment characteristics of the patients and the site and stage of the tumour, according to the $4^{\text {th }}$ edition of the TNM used at that time [12], are reported in table 1 and table 2 . The T2 patients were submitted to this protocol owing to general conditions that contraindicated surgery or because they refused surgery.

Table I: Pre-treatment characteristics of the patients

\begin{tabular}{lc}
\hline Patient characteristics & Number \\
\hline Sex & 43 \\
Males & 3 \\
Females & $39-75$ \\
Age & 58.3 \\
Range & 42.1 \\
Mean & $>80$ \\
Median & \\
Performance status & 7 \\
(Karnofsky scale) & 15 \\
Tumor stage & 24 \\
III & 28 \\
IV & 28 \\
Primary tumour site & 3 \\
Oral cavity & 1 \\
Oropharynx & 4 \\
Hypopharynx & 1 \\
Nasopharynx & 2 \\
Oral cavity + Oropharynx & 2 \\
\hline
\end{tabular}


Table 2: Pre-treatment tumour $(\mathrm{T})$ and lymph node $(\mathrm{N})$ staging

\begin{tabular}{cccccccc}
\hline & N0 & NI & N2a & N2b & N2c & N3 & TOTAL \\
\hline T2 & 8 & 4 & 5 & 1 & 1 & 19 \\
T3 & 9 & 5 & 3 & 1 & 1 & 19 \\
T4 & 1 & 4 & 8 & 2 & 1 & 8 \\
TOTAL & 18 & 13 & 4 & 3 & 0 & 46 \\
\hline
\end{tabular}

Patients were required to sign informed consent approved by the Ethical Committee of the Institution.

\section{Pre-treatment evaluation}

Patients underwent a complete clinical and laboratory examination including measurement of haematologic (complete blood cell and platelet count, prothrombin time, partial thromboplastin time), hepatic (blood bilirubin, transaminases and alkaline phosphatase level)and renal (blood urea nitrogen, creatinine and electrolytes level, urinanalysis, 24 hours creatinine clearance) parameters, as well as general metabolic functions (blood glucose and lipids level), chest radiography and cervical vessel ultrasonography. The disease was staged by physical examination, panendoscopic examination, biopsy, CT scan and/or MRI.

\section{Treatment protocol}

All patients received intra-arterial (i.a.) infusions of carboplatin, dissolved in $80 \mathrm{~mL}$ of $0,9 \%$ saline and infused over 10-15 min by a battery-operated pump (Medrad Mark 5 Plus) with an infusion velocity of $6-8 \mathrm{~mL} / \mathrm{min}$. An angiographic catheter (Glidecath Radicofocus 5F Terumo) was introduced percutaneously under local anesthesia $(10 \mathrm{~mL}$ of $2 \%$ lidocaine solution) into the common femoral artery, according to the Seldinger technique. The catheter tip was placed in the external carotid artery, under radiographic control. Diagnostic transfemoral carotid arteriography was performed and the most suitable branch of the artery, providing the main blood supply to the tumour, was selectively catheterised. The catheter was removed immediately after the infusion. A simultaneous intravenous (i.v.) infusion of $250 \mathrm{~mL}$ of $0,9 \%$ saline was performed. Dexamethasone (4 mg) and ondansetron (1 g) dissolved in $100 \mathrm{~mL}$ of $0,9 \%$ saline were administered i.v. 1 hour before the infusion. All patients received three cycles of intra-arterial chemotherapy, administered every two weeks. The planned dose of carboplatin per cycle was $300 \mathrm{mg} / \mathrm{m}^{2}$ for the first cohort (3 cases) and $325 \mathrm{mg} / \mathrm{m}^{2}$ for the second cohort ( 3 cases); subsequently, as no significant toxic effects were observed, the remaining patients (40 cases) received a dose of $350 \mathrm{mg} / \mathrm{m}^{2}$ of carboplatin per cycle. Two weeks after completing chemotherapy, patients were restaged and subsequent treatment was decided upon on the basis of their objective response. Complete responders or partial responders had radiotherapy; non-responders with resectable disease underwent surgery on the tumor site and/or on the neck nodal metastases followed by radiotherapy; non-responders with unresectable disease underwent palliative radiotherapy. Radiation was delivered using conventional fractionation (1,8 - 2 Gy per fraction, five days per week). The total dose was $66-70$ Gy for PTVI (planning target volume including tumour and encompassing all clinically positive nodes) and 50-56 Gy for PTVII (planning target volume including all anatomical regions at risk of subclinical malignant disease).

\section{Treatment evaluation}

Toxicity was evaluated according to WHO criteria [13] 24 hrs after each drug administration. This included: assessment of haematologic, hepatic and renal parameters; assessment of gastrointestinal, otoneurological, cardiac and respiratory function, and the search for signs and symptoms of local toxicity. Response to therapy was assessed two weeks after the last administration of the drug by physical examination, endoscopy, imaging (CT scan and/or MRI) and biopsy, when necessary. A complete response (CR) was defined as the complete disappearance of all demonstrable lesions. A partial response (PR) was defined as a decrease of $50 \%$ or greater in the sum of the products of the largest perpendicular diameters of all measurable lesions. No response (NR) was defined as a regression of $<50 \%$ in total tumour size or as stable or progressive disease.

\section{Statistical analysis}

The primary efficacy end point was overall survival (i.e, death as a result of any cause). Survival was measured from the start of treatment date to the date of death or the date when the patient was last known to be alive. Yearly estimates were calculated using the Kaplan-Meier method. Secondary efficacy end points were CR rates, loco-regional tumour control and disease-free survival. Time to locoregional failure was measured from the start of treatment date and the date of disease relapse, the date of diseaserelated death, or the date when the patient was last known to be alive and disease-free. Loco-regional failure, distant 
Table 3: Toxicity from chemotherapy

\begin{tabular}{ccc}
\hline Type of toxicity & Grade & N. of patients \\
\hline Neutropenia & 2 & 2 \\
Thrombocytopenia & 1 & 2 \\
Hyperbilirubinemia & 4 & 1 \\
Peripheral neuropathy & 3 & 1 \\
Alopecia & $1-3$ & 1 \\
Stomatitis & $2-3$ & 2 \\
Skin rash & 3 & 1 \\
\hline
\end{tabular}

metastases, or disease-related death were all considered failures for disease-free survival. Yearly disease-free survival rates were estimated with the Kaplan-Meier method.

Factors influencing overall and disease-free survival were analyzed and compared by the log-rank test. P values less than 0.05 were considered significant.

\section{Results}

\section{Feasibility}

One hundred and thirty eight super-selective transfemoral intra-arterial infusions of carboplatin were performed with no significant complications.

\section{Toxicity}

Carboplatin treatment was well-tolerated by the majority of the patients. No instances of gastrointestinal (nausea, vomiting, diarrhoea), renal, cardiac, respiratory and otoneurological toxicity were reported. Cases of systemic and local toxicity are listed in table 3.

\section{Response rate}

After completing chemotherapy, 16 patients had a CR (35\%), 20 patients had a PR (43\%) and 10 patients had NR $(22 \%)$. The 36 patients with a complete or partial response underwent radiotherapy, after which 26 patients achieved a complete response. Among the other 10 patients with persistent or recurrent disease in the neck, 5 underwent neck dissection and achieved a complete response and 5 refused any further treatment. Seven of the 10 NR patients underwent neck and primary site surgery, followed by radiotherapy. After this treatment, all 7 patients achieved a complete response. Of the remaining 3 non-responding patients, one refused any further treatment and two had palliative radiotherapy. Upon completing all treatments, 38/46 patients (83\%) achieved a complete response: the 26 patients (CR) treated with induction chemotherapy and radiotherapy; the 5 (PR) treated with induction chemotherapy, radiotherapy and neck surgery and the 7 (NR) treated with induction chemotherapy, primary site and neck surgery and radiotherapy. (Fig. 1)

\section{Control of disease and survival}

No patients were lost to follow-up. The median follow-up time, measured from the start of induction chemotherapy, was 51.3 months (range: 5 to 136 months).

The current status of the patients is as follows: after completing the treatment protocol, the 8 patients with persistent or recurrent disease ( 3 from the NR group and 5 from the PR group) have died of the disease; 13 of the $38 \mathrm{CR}$ patients are alive and disease-free, 3 have died of distant metastases ( 2 pulmonary and 1 hepatic) and 3 due to a second primary tumour (2 lung cancers and 1 renal can-

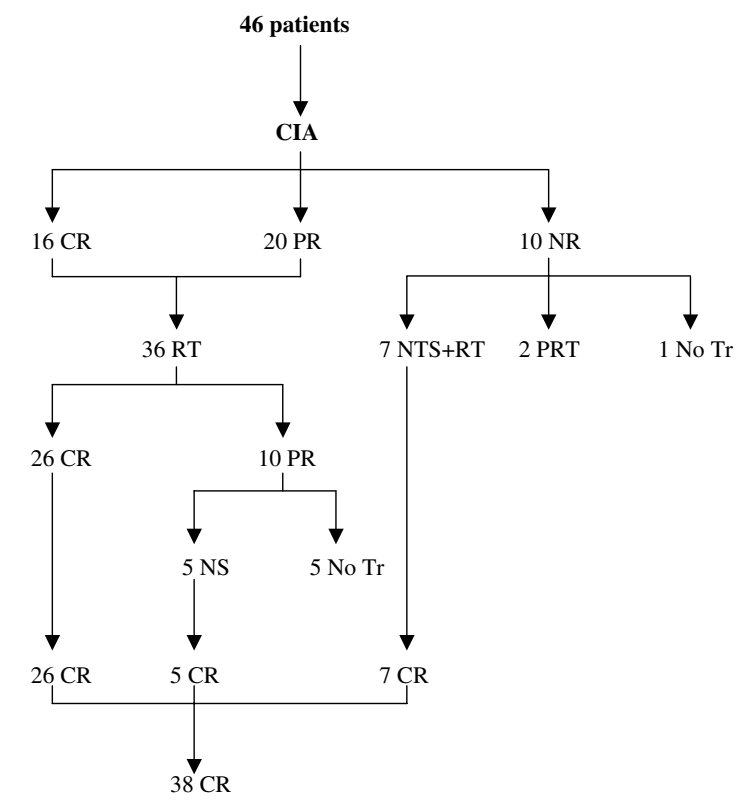

\section{Figure I}

Locoregional response after completion of treatment protocol $(\mathrm{CIA}=$ intra-arterial chemotherapy; $\mathrm{CR}=$ complete response; $\mathrm{PR}=$ partial response; $\mathrm{NR}=$ no response; $\mathrm{RT}=$ radiotherapy; NS = neck surgery; NTS = primary and neck surgery; $\mathrm{PRT}=$ palliative radiotherapy; $\mathrm{No} \mathrm{Tr}=$ no treatment). 
cer), which occurred after a mean period of 29 months. The tumour recurred in 19 patients after a mean period of 25 months; 9 on the neck and 10 at the primary site. Five of the 9 patients with tumour recurrence on the neck underwent neck dissection; of these 2 are still alive and disease-free and 3 have died due to the persistence of the tumour; the other 4 received systemic chemotherapy. Of these 1 is alive and free of disease while 3 have died of the disease. Of the 10 patients in whom tumours recurred at the primary site, 5 underwent palliative systemic chemotherapy and died of the disease, 2 received only palliative medical treatment and died of the disease and 3 underwent salvage surgery. Of these, 2 are alive and disease-free and 1 has died of pulmonary metastases. In conclusion, after a 5-year follow-up, 18 patients (39\%) are alive and disease-free, $3(6,5 \%)$ have died due to a second primary tumour and $25(54,5 \%)$ have died of the disease (Fig. 2).

The percentage of organ preservation was $83 \%$, as 8 of the 46 patients underwent salvage surgery at the tumour site.

The probability of five-year overall survival was 50\% (Fig. 3 ). The main factor influencing overall survival was the response to induction chemotherapy: CR patients showed statistically significant better survival rates compared to

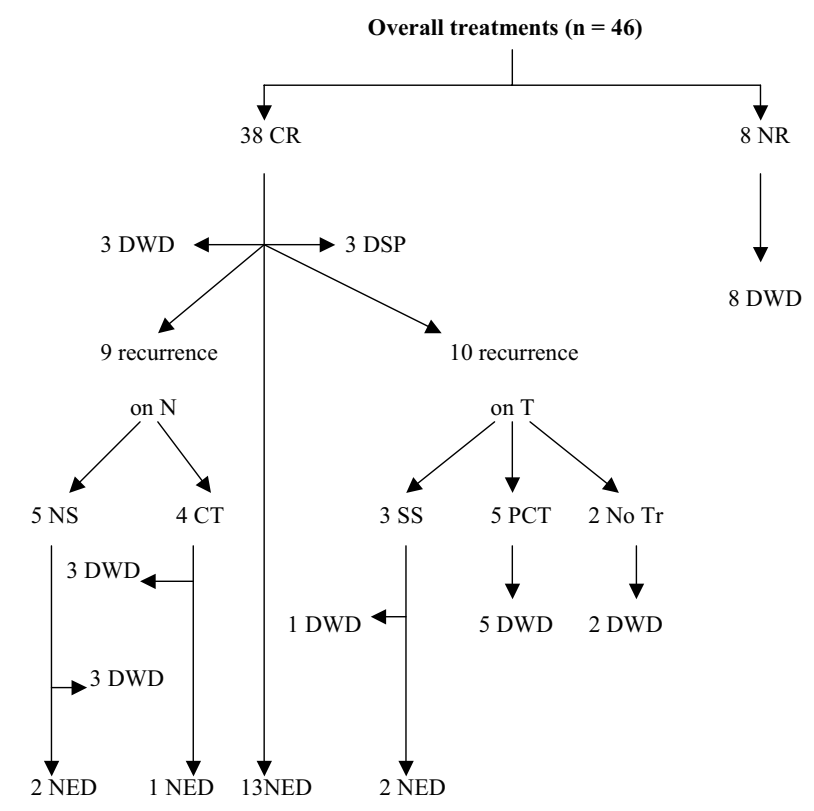

Figure 2

Clinical status of the patients at the end of follow-up $(C R=$ complete response; $P R=$ partial response; NED = no evidence of disease; DWD = death due to disease; $D S P=$ death due to second primary tumour; NS = neck surgery; $\mathrm{SS}=$ salvage surgery; $\mathrm{CT}=$ systemic chemotherapy; $\mathrm{PCT}=$ palliative systemic chemotherapy; No $\mathrm{Tr}=$ no treatment).

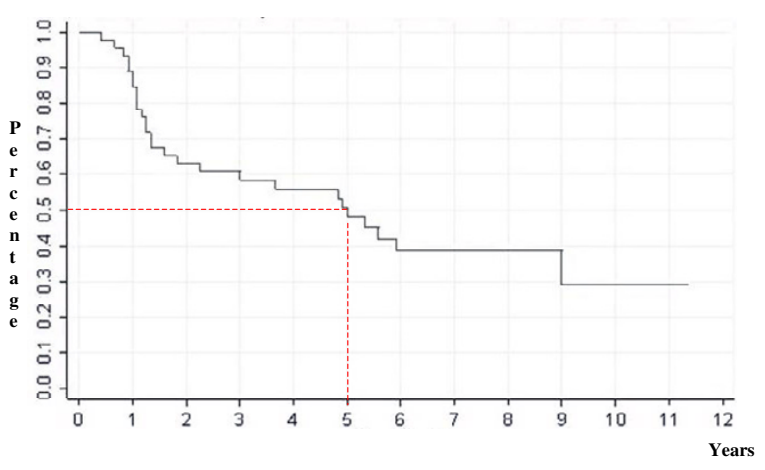

Figure 3

Overall survival of 46 patients.

PR or NR patients, even if $12(40 \%)$ of these patients were successfully submitted to salvage surgery (Fig. 4).

Five-year disease-free survival, calculated on the 38 patients who were free of disease after completing all treatments, was $49,8 \%$ (Fig. 5). In this case, the main factor influencing disease recurrence or the appearance of distant metastases was patients' neck status: patients with no metastatic lymph-nodes or patients whose metastatic lymph-nodes regressed completely after induction chemotherapy had a better disease-free survival rate than patients with positive nodes who had a partial or no response to chemotherapy (Fig. 6).

\section{Discussion}

Our present data confirmed the evidence that regional chemotherapy by superselective transfemoral infusion of high doses of platinum compounds is feasible and that, with the cooperation of an experienced interventional radiologist, can be easily reproduced in a multi-institu-

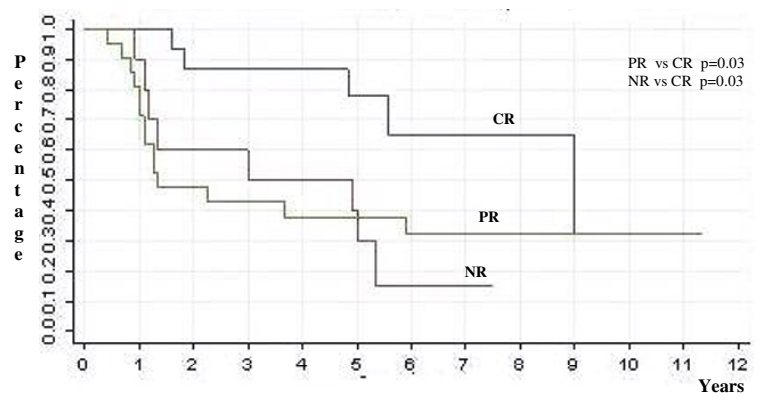

Figure 4

Overall survival according to the response to induction chemotherapy $(C R=$ complete response; $P R=$ partial response; $\mathrm{NR}=$ no response). 


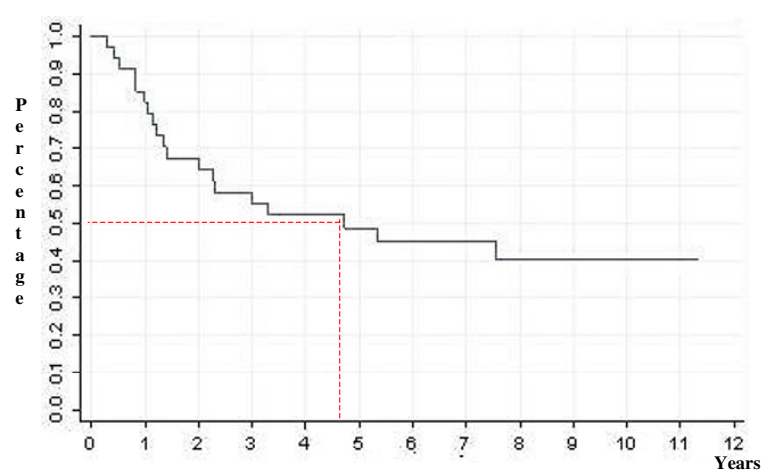

Figure 5

Disease-free survival of 38 patients.

tional setting, with minimal procedural complications [9,11,14-16].

The advantage of carboplatin is that it has an antitumour activity comparable with that of cisplatin but a toxicology profile with lower renal and gastrointestinal effects [10,17-19]. We administered far higher doses of carboplatin than those used by Vieitez et al. [15], according to our preliminary phase I study [20] that led us escalating the cycle doses of carboplatin from $300 \mathrm{mg} / \mathrm{m} 2$ to $350 \mathrm{mg} /$ $\mathrm{m} 2$. These doses were comparable to the cisplatin "decadoses" used by Robbins et al. [21-24], without relying on simultaneous i.v. treatment with sodium thiosulphate to achieve systemic neutralisation of the platinum compound.

Systemic toxicity was low in our study population: all patients completed the three cycles of chemotherapy with no chemotherapy-related deaths, only 4 cases of moderate (grade 1-2) hematologic toxicity, and one case each of high (grade 3-4) hepatic and neurological toxicity. Local

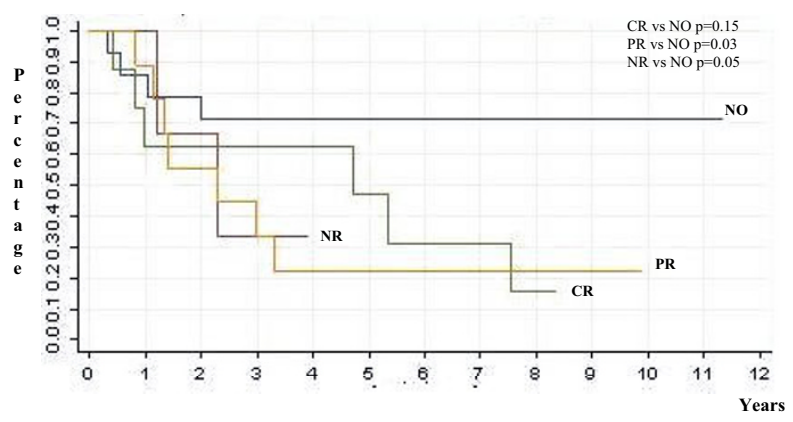

Figure 6

Disease-free survival according to neck status (NO = no lymphnodes at diagnosis; $C R=$ complete response; $P R=$ partial response; $\mathrm{NR}=$ no response). toxicity occurred in some cases, mainly at the level of skin and oral mucosa, but of short duration and of low/moderate degree. The higher systemic toxicity observed in other studies based on the same intra-arterial chemotherapy approach could be due to the different activity/toxicity profile of cisplatin versus carboplatin or to the potentiation of toxic effects in concurrent chemotherapyradiotherapy regimens.

With respect to a classic neoadjuvant intravenous chemotherapy protocol (Al Sarraf) our protocol presents the advantage of a reduced systemic toxicity and of a reduced duration (one day vs. three days administration, two weeks vs. three weeks interval), with a significant improvement of patient's compliance.

A true neoadjuvant intra-arterial chemotherapy approach for head and neck cancer treatment is rare and the results of many trials are difficult to compare, owing to a number of methodological differences [16]. Limiting our comparison to the latest studies conducted with platinum compounds, our response rates at the end of induction chemotherapy (CR 35\% and PR 43\%) are similar to those observed by Kovacs [16] (CR 38\% and PR 31\%) and lower than those observed by Wilson et al [25] (CR 68\% and PR $23 \%)$.

Our five-year overall and disease-free survival rates (50\% and $49,8 \%$ ) may be compared with those observed by Kovacs [16] (77\% and 59\%). Even if concurrent chemoradiation treatments seem to achieve the best overall survival rate [1], our results (50\% and $49,8 \%)$ are similar to those obtained with the RADPLAT protocol $(38,8 \%$ and $53,6 \%)[26]$.

According to our previous results [11] and the opinion of other authors $[2,27]$, we consider the response to induction chemotherapy to be an important prognostic factor which may determine the sequence and timing of further planned definitive therapy: patients with a lesser response will require total surgical resection of the disease or the remaining disease, followed by post-surgical radiation therapy; patients with a good response may avoid surgery and be treated with radiotherapy alone. Therefore, on the basis of the evaluation made after induction chemotherapy, we adopted a flexible protocol, based not only on the patient's compliance and general conditions, but mainly on their response to neoadjuvant chemotherapy. A similar but less customized multimodality treatment has been followed by Kovacs et al. [28] in a large series of oral and oropharyngeal cancer patients.

\section{Conclusion}

Our present experience suggests that in the treatment of head and neck cancer, superselective intra-arterial infu- 
sion with high doses of carboplatin delivered directly to the tumour bed is a safe and well-tolerated technique with no major systemic or local toxic side effects. It proves once again that, compared to other protocols (e.g. concurrent chemoradiotherapy), systemic or intra-arterial induction chemotherapy, does not have a significant advantage in terms of overall or disease-free survival. However, in some patients with bulky head and neck tumours, this technique, adopted as part of a first-line multidisciplinary approach, can achieve a high response rate, which may have a prognostic significance. This is a prospective study of a cohort of patients treated in a unique fashion in which carboplatin was given as an intra-arterial infusion. Those patients who had a major response were than treated with radiation therapy only. Non-responders were treated with surgery. The protocol introduces a novel algorithm for management of patients with advanced head and neck cancer. It is designed to triage patients who are sensitive to chemotherapy to non-surgical regimen whereas those who are not sensitive are treated with surgery.

\section{Competing interests}

The author(s) declare that they have no competing interests.

\section{Authors' contributions}

GB and PG prepared and edited the manuscript. MB, FC, GB and FZ were involved in revising results and in preparation of the different topics of the manuscript (surgery, radiotherapy, chemotherapy and infusional technique). CT performed the statistical analysis. EM gave the final approval of the version of the manuscript.

\section{Acknowledgements}

The authors gratefully acknowledge Dr. Th. Robbins for the revision of the manuscript.

\section{References}

I. Pignon JP, Bourhis J, Domenge C, Designe L: Chemotherapy added to locoregional treatment for head and neck squamous-cell carcinoma: three meta-analyses of updated individual data. Lancet 2000, 355:949-955.

2. Al-Sarraf M: Treatment of locally advanced head and neck cancer: historical and critical review. Cancer Control 2002, 9:387-399.

3. Argiris A: Update on chemoradiotherapy for head and neck cancer. Curr Opin Oncol 2002, 14:323-329.

4. The Department of Veterans Affairs Laryngeal Cancer Study Group: Induction chemotherapy plus radiation compared with surgery plus radiation in patients with advanced laryngeal cancer. N Engl J Med I99I, 324: |685-1690.

5. Domenge C, Hill C, Lefebvre JL, De Raucourt D, Rhein B, Wibault P, Marandas P, Coche-Dequeant B, Stromboni-Luboinski M, SanchoGarnier H, Luboinski B: Randomized trial of neoadjuvant chemotherapy in oropharyngeal carcinoma. Br J Cancer 2002, 83:1594-1598.

6. Kim S, Wu HG, Heo DS, Kim KH, Sung MW, Park Cl: Advanced hypopharyngeal carcinoma treatment results according to treatment modalities. Head Neck 200I, 23:7I3-7I7.

7. Vokes EE, Stenson K, Rosen FR, Kies MS, Rademaker AW, Witt ME, Brockstein BE, List MA, Fung BB, Portugal L, Mittal BB, Pelzer H,
Weichselbaum RR, Haraf DJ: Weekly carboplatin and paclitaxel followed by concomitant paclitaxel, fluorouracil, and hydroxyurea chemoradiotherapy: curative and organ-preserving therapy for advanced head and neck cancer. J Clin Oncol 2003, $21: 320-326$.

8. Altundag O, Gullu I, Altundag K, Yalcin S, Ozyar E, Cengia M, Akyol F, Yucel T, Hosal S, Sozeri B: Induction chemotherapy with cisplatin and 5-fluorouracil followed by chemoradiotherapy or radiotherapy alone in the treatment of locoregionally advanced resectable cancers of the larynx and hypopharynx: results of single-center study of $\mathbf{4 5}$ patients. Head Neck 2005 , 27:|15-2I.

9. Robbins KT, Kumar P, Harris J, McCulloch T, Cmelak A, Sofferman R, Levine P, Weisman R, Wilson W, Weymuller E, Fu K: Supradose intra-arterial cisplatin and concurrent radiation therapy for the treatment of stage IV head and neck squamous cell carcinoma is feasible and efficacious in multi-institutional setting: results of radiation therapy oncology group trial 9615 . J Clin Oncol 2005, 23: |447-1454.

10. Volling P, Schroder M, Rauschning W, Achterrath W, Stennert E: Carboplatin. The better platinum in head and neck cancer? Arch Otolaryngol Head Neck Surg 1989, I I 5:695-698.

II. Benazzo M, Caracciolo G, Zappoli F, Bernardo G, Mira E: Induction chemotherapy by superselective intra-arterial high-dose carboplatin infusion for head and neck cancer. Eur Arch Otorhinolaryngol 2000, 257:279-282.

12. International Union Against Cancer: TNM Classification of malignant tumours. Fourth edition. New York: Wiley-Liss; 1987.

13. World Health Organization: WHO Handbook for Reporting Results of Cancer Treatment. WHO offset publication. Geneva: World Health Organization; 1979:48.

14. Robbins KT, Kumar P, Harris J, McCulloch T, Cmelak A, Sofferman R, Levine P, Weisman R, Wilson W, Weymuller E, Fu K: Supradose intra-arterial cisplatin and concurrent radiation therapy for the treatment of stage IV head and neck squamous cell carcinoma is feasible and efficacious in a multi-institutional setting: results if radiation therapy oncology group trial 9615 . J Clin Oncol 2005, 23: | 447-I454.

15. Vieitez JM, Bilbao JI, Hidalgo OF, Martin S, Manzano RG, Tangco E: Intra-arterial chemotherapy with carboplatin and 5-fluorouracil in epidermoid cancer of the oropharynx and oral cavity. Reg Cancer Treat 1991, 4:152-I55.

16. Kovacs AF: Intra-arterial induction high-dose chemotherapy with cisplatin for oral and oropharyngeal cancer: long-term results. Br J Cancer 2004, 90: 1323-1328.

17. Calvert AH, Harland SJ, Newell DR, Siddik ZH, Harrap KR: Phase I studies with carboplatin at the Royal Marsden Hospital. Cancer Treat Rev 1985, I 2:5I-57.

18. Foster BJ, Clagget-Carr K, Leyland-Jones B, Hoth D: Results of NCIsponsored phase I trials with carboplatin. Cancer Treat Rev 1985, 1 2:43-49.

19. Rose WC, Schuring JE: Preclinical antitumor and toxicologic profile of carboplatin. Cancer Treat Rev 1985, I2:1-19.

20. Benazzo M, Bernardo G, Corbella F, Danova M, Lanza L, Zappoli F, Uggetti C, Mira E: La chemioterapia intraarteriosa neo-adiuvante superselettiva con carboplatino ad alte dosi nei tumori avanzati cervico-facciali. Acta Otorhinol Ital 1996, I 6:30-34.

21. Robbins KT, Storniolo M, Kerber C, Seagren S, Berson A, Howell SB: Rapid superselective high-dose cisplatin infusion for advanced head and neck malignancies. Head Neck 1992, |4:364-37|.

22. Robbins KT, Storniolo AM, Kerber C, Vicario D, Seagren S, Shea M, Hanchett C, Los G, Howell SB: Phase I study of highly selective supradose cisplatin infusion for advanced head and neck cancer. J Clin Oncol 1994, I 2:21 I3-21 20.

23. Robbins KT, Fontanesi J, Wong FS, Vicario D, Seagren S, Kumar $P$, Weisman R, Pellitteri P, Thomas JR, Flick P, Palmer R, Weir AB 3rd, Kerber C, Murry T, Ferguson R, Los G, Orloff L, Howell SB: A novel organ preservation protocol for advanced carcinoma of the larynx and pharynx. Arch Otolaryngol Head Neck Surg 1996, I 22:853-857.

24. Robbins KT, Kumar P, Regine WF, Wong FS, Weir AB 3rd, Flick $P$, Kun LE, Palmer R, Murry T, Fontanesi J, Ferguson R, Thomas R, Harstell WF, Paig CU, Salazar G, Norfleet L, Hanchett CB, Harrington $V$, Neill HB: Efficacy of targeted supradose cisplatin and concomitant radiation therapy for advanced head and neck can- 
cer: the Memphis experience. Int J Radiat Oncol Biol Phys 1997, 38:263-27I.

25. Wilson WR, Siegel RS, Harisiadis LA, Davis DO, Nguyen HH, Bank WO: High-dose intra-arterial cisplatin therapy followed by radiation therapy for advanced squamous cell carcinoma of the head and neck. Arch Otolaryngol Head Neck Surg 200I, I 27:809-8|2.

26. Robbins KT, Kumar P, Wong FSH, Harstell WF, Flick P, Palmer R, Weir AB 3rd, Neill HB, Murry T, Ferguson R, Hanchett C, Vieira F, Bush A, Howell SB: Targeted chemoradiation for advanced head and neck cancer: analysis of 213 patients. Head Neck 2000, 22:687-693.

27. Kovacs AF: Intra-arterial chemotherapy and chemoembolization in head neck cancer. Establishment as a neoadjuvant routine method. Cancer Ther 2003, I:I-9.

28. Kovacs AF, Schiemann M, Turowski B: Combined modality treatment of oral and oropharyngeal cancer including neoadiuvant intra-arterial cisplatin and radical surgery followed by concurrent radiation and chemotherapy with weekly docebaxel - three year results of a pilot study. I Cranio-Maxillofac Surg 2002, 30:112-120.

\section{Pre-publication history}

The pre-publication history for this paper can be accessed here:

http://www.biomedcentral.com/1471-2407/7/62/prepub

Publish with Bio Med Central and every scientist can read your work free of charge

"BioMed Central will be the most significant development for disseminating the results of biomedical research in our lifetime. "

Sir Paul Nurse, Cancer Research UK

Your research papers will be:

- available free of charge to the entire biomedical community

- peer reviewed and published immediately upon acceptance

- cited in PubMed and archived on PubMed Central

- yours - you keep the copyright 